\title{
Epithelial Ovarian Cancer-Cancer Institute (WIA) Experience
}

\author{
V. SRIDEVI, R. RAJIV RAJENDRENATH, V.SHANTA
}

\section{INTRODUCTION}

The Cancer Institute (WIA) is a regional cancer centre that was started in 1954.

We have been the pioneers of neo-adjuvant therapy- Radiotherapy, Chemotherapy and Concurrent chemo-radiotherapy in various sites such as Head and Neck cancers, breast and ovarian cancers. There has been an increase in the incidence of EOC over the last few years .

\section{METHODS}

This is a retrospective analysis of 636 patients with EOC who were treated from 1985 to 1999 at the institute. A majority of these were advanced stage and received neo-adjuvant therapy and they were re-assessed for Interval cytoreduction after 3 or 4 cycles. Patients were initiated on NAC after confirmation of malignancy by ascitic fluid cytology, Pouch of Douglas aspiration or a FNAC- Transabdominal or transvaginal. Patients with clinical or radiological evidence of advanced diseaseperitoneal, omental, pelvic nodules and moderate ascites were all initiated on NAC.At the completion of the $3 \mathrm{rd}$ chemotherapy they were reassessed. Patients who showed regression - clinical, radiological and / or biochemical were taken for Interval Debulking Surgery (IDS). Those patients who showed evidence of progression were either continued on the same chemotherapy or changed to oral or salvage chemotherapy. After the IDS ,patients completed the remaining cycles of chemotherapy - to a total of 6 cycles and 1 cycle Intra-peritoneal chemotherapy was given to most patients who had optimal cytoreduction.
The patients who had surgery outside and then came to the institute were initiated on chemotherapy. Primary Staging Laparotomy was not done in this group. Those patients with resectable disease or those patients in whom the hysterectomy and contralateral oophorectomy were not done initially underwent interval debulking surgery after 3 cycles of chemotherapy.

Patients who had early stage disease - with no evidence of pelvic nodules or ascites underwent Primary cytoreduction and then adjuvant chemotherapy .

Patients were on follow up with markers and clinical examination every 3 months and annual USG . Patients with relapse were given chemotherapy based on the interval from the last therapy.

\section{RESULTS}

The stage distribution did not show any major change over the 15 year period with stage III disease accounting for the majority. The 5 and 10 year survival of the patients who had surgery at Cancer Institute for stage III disease was 42.8 and $38.9 \%$ respectively. However if the surgery was not done the survival dropped to 21.8 and $18.6 \%$ respectively. In stage I the 5 and 10 year survival was 82.2 and 74.5 , whereas in stage II the same was 55.8 for both the periods.

\section{CONCLUSION}

EOC has shown an increasing incidence and it continues to be a major cause of mortality among women. Newer methods of early detection and better therapeutic strategies are required to improve the outlook in EOC 